\title{
Distances on hills look farther than distances on flat ground: Evidence from converging measures
}

\author{
Nathan L. Tenhundfeld ${ }^{1}$. Jessica K. Witt $^{1}$
}

Published online: 10 March 2017

(C) The Psychonomic Society, Inc. 2017

\begin{abstract}
Distances on hills are judged as farther than when the same distance is presented on the flat ground. The hypothesized reason for this difference is because perception is influenced by the increased effort required to walk up a hill than to walk the same distance on flat ground. Alternatively, distances presented up a hill might be judged as farther for other, nonperceptual reasons such as bias from demand characteristics. To test whether distances on hills are perceived as farther or are merely judged as farther, we used a variety of measures, including visual matching and blindwalking tasks, and found similar effects across all measures. This convergence is consistent with a perceptual explanation. Second, we mined our data with the goal of making recommendations for future research on this paradigm. Although all of the perceptual measures used showed similar effects, visual matching was the only measure that had good intrasubject reliability. We recommend that future research on this action-specific effect could use any measure unless the research is geared towards individual differences, in which case, only the visual matching measure of perceived distance should be used.
\end{abstract}

Keywords Perception action · Distance perception · Embodied perception $\cdot$ Reliability

Distances presented up a hill are judged as farther than distances on the flat ground (Stefanucci, Proffitt, Banton, \& Epstein, 2005). This distance-on-hill effect is argued to be a perceptual bias that is a function of the energetic cost

Nathan L. Tenhundfeld

Nate.Tenhundfeld@colostate.edu

1 Colorado State University, Behavioral Sciences Building, Fort Collins, CO 80523, USA associated with traversing a given distance on a hill. The suggestion is that because it takes more effort to walk a fixed distance up a hill than on flat ground, the perceptual system accounts for this increase in effort by distorting how far that distance looks (Proffitt, 2006). This action-specific hypothesis has been supported by other research showing that wearing a heavy backpack leads to greater distance estimations (Proffitt, Stefanucci, Banton, \& Epstein, 2003), as can other actions such as throwing a heavy ball when compared to throwing a lighter one (Witt, Proffitt, \& Epstein, 2004), or jumping across gaps while wearing ankle weights (Lessard, Linkenauger, \& Proffitt, 2009). If these effects are perceptual in nature, it would mean that perception relies on a synthesis of both visual and nonvisual information, such as energetics and fatigue (Bhalla \& Proffitt, 1999).

Questions remain, however, as to whether effects such as the distance-on-hills effect are truly perceptual (Firestone \& Scholl, 2016; Philbeck \& Witt, 2015; Witt, in press). The observed differences in perceptual judgments could be due to effects on postperceptual processes instead. For example, a potential alternative explanation is that judging distances on a hill versus on flat ground creates a demand characteristic to respond that the distances up the hill are farther, even if they are perceived to be the same as the distances on flat ground. Perhaps participants are conforming to what they believe to be the direction of the predicted effect (Durgin et al., 2009) and this accounts for the differences in the responses rather than genuine differences in perception. In the case of the distanceon-hill effect, only verbal estimates of distance have been used (Stefanucci et al., 2005). Although verbal estimates can be an effective measure of perceived distance, they are also prone to many kinds of biases (Pagano \& Isenhower, 2008; Poulton, 1979).

There are many strategies to address whether or not a given effect is perceptual. One strategy is to use a visual matching 
task for which participants judge when two distances appear to be the same. In a typical visual matching task, participants instruct an experimenter to adjust a reference marker so that the distance between the participant and the reference marker is the same as the egocentric distance to the target. These visual matching tasks have several advantages over verbal estimates. First, most people do not have extensive experience with labeling distances in feet and inches, and can struggle to put a numeric label on a distance. As noted by Woods, Philbeck, and Danoff (2009), people often say that their distance perception is terrible but in doing so, "people directly equate 'poor distance perception' with their sense of unfamiliarity with assigning numbers to distances" (p. 1104). Visual matching tasks, in contrast, do not require this difficult assignment. Instead, participants can simply look at two distances and determine whether they are the same or different. In addition, as long as the experiment takes place in an environment devoid of landmarks, visual matching tasks are less likely to be biased by memory of previous responses because participants are not as easily able to compare their estimates to previous estimates. However, visual matching tasks are not without their own biases. Specifically, when the comparison distance is initially short, the final comparison distance tends be shorter than when the initial comparison distance is far (e.g., Witt et al., 2004). Researchers can overcome this inherent bias by ensuring equal number of trials or groups with each of the two initial distances so that the two biases (shorter estimates with a closer starting point and farther estimates with a farther starting point) cancel out each other when calculating the mean response across trials or groups.

A second strategy is to use action-based measures of perception such as blindwalking to assess perceived distance (Loomis \& Philbeck, 2008). In blindwalking tasks, participants close their eyes and walk the perceived distance, stopping when they have reached the target's location or have walked the same distance as the target distance but in a different direction. The distance walked serves as a measure of their perception such that if they perceive the target as farther away, they should blindwalk farther. Blindwalking has an advantage as a measure of perception because of its undeniable accuracy (Loomis, Da Silva, Fujita, \& Fukusima, 1992). In addition, blindwalking does not require any kind of verbal judgment whatsoever. Because it is an action measure, rather than an explicit judgment measure, blindwalking also has the advantage that it is not likely to be biased by different interpretations across instructions. When asked to verbally estimate the distance to a target, there are several possible interpretations. Participants could estimate based on cognitive knowledge of the distance to the target, their perception of the distance to the target, or they could misattribute feelings of closeness when estimating distance (Woods et al., 2009). In contrast, it is unlikely that blindwalking to a target has as many possible interpretations.
Despite the advantages of action-based measures, there is also concern as to whether action measures are driven by a different source of visual information, namely, information processed in the dorsal pathway. According to the theory of two visual streams (cf. Goodale \& Milner, 1992), conscious perception is driven by processing in the ventral pathway, and can be assessed using measures such as verbal reports and visual matching, but is prone to biases (as demonstrated by various illusions such as the Müller-Lyer illusion). In contrast, the processing in the dorsal pathway is not prone to biases, and it is this information that is used to control visually guided actions. Previous research on hill slant perception demonstrated that verbal reports and visual matching tasks revealed effects of fatigue and energetics, but the haptic-based measure of manually adjusting a board to match the slant of the hill was unaffected (Bhalla \& Proffitt, 1999). However, it is important to note that not all action-based measures are privy to dorsal stream processing. In particular, the only actions that are guided by information in the dorsal stream are actions that are closed-loop, meaning that the target is viewed throughout the duration of the controlled action (Heath, Rival, Westwood, \& Neely, 2005). In the current experiments, the action-based measure is blindwalking, which is an open-loop action because the target is not visible during the action. Thus, it should be expected that if a factor such as energetics influences consciously perceived distance to targets, this effect should be apparent in the measure of blindwalking. If both verbal estimates and visual matching show the distance-onhill effect, but the measure of blindwalking had not, the data would cast doubt on a perceptual interpretation of the distance-on-hill effect.

A third strategy to discern between perceptual and postperceptual effects is to examine convergence across a variety of measures. This strategy has been promoted previously by researchers who studied nonvisual biases in distance perception (Foley, 1977; Gogel, 1990; Loomis, 2016; Philbeck \& Loomis, 1997). The idea is that if an effect is truly perceptual, the pattern of responses that characterizes the effect should emerge across multiple measures of perception. In contrast, if an effect is only apparent in verbal estimates but not in other kinds of measures like visual matching and action-based measures, this would be strong evidence in favor of a postperceptual, judgment-based effect.

Another potential strategy could be to try to implement an illusion-nulling technique. This strategy is often used in motion perception research, where it is called the motion nulling technique. The idea is that objects are presented that are moving in the opposite direction as the perceived motion so that the physical motion and the perceived motion cancel out each other and the resulting perception is that the target appears to be stationary. Here, we applied this idea to the distance-onhills effect by presenting a secondary illusion that was theorized to have the opposite effect on perceived distance, namely 
make the target appear closer. We tested whether the two illusions would cancel each other out. To preview our results, this strategy was ineffective because the secondary illusion did not impact perceived distance and thus was ineffective at nulling the distance-on-hills effect. Given that this strategy has not been promoted outside of the motion perception literature, we did not pursue it further beyond the initial attempt.

One last strategy used here is a survey to determine the degree to which participants can predict the direction of the effect. If this effect is due to response bias, we should expect that the number of participants who are able to guess the direction of the effect is greater than chance. If participants are able to accurately determine the predicted direction of the effect at rates higher than chance, future studies should aim to exclude such participants with questionnaires in the future. However, if participants, as a whole, are unable to predict the direction of the distance-on-hill effect at a rate greater than chance, not only will this suggest that the effect itself is not due to response bias, but additionally will render the need for postexperimental surveys obsolete when using this paradigm.

The primary purpose of the current experiments was to use these aforementioned strategies to assess whether or not the distance-on-hills effect is perceptual. While Stefanucci et al. (2005) were able to find convergence across real and virtual environments, the concerns regarding the use of only verbal estimates prevails.

A second goal of the current research was to make recommendations for future research. If the new measures do not reveal the effect, or convergence is not achieved, the recommendation would be that the distance-on-hills effect is not a genuinely perceptual effect. However, if the data are consistent with a perceptual explanation, future research would still be necessary to address questions concerning other nonperceptual explanations (such as those raised by Firestone \& Scholl, 2016) as well as the underlying mechanisms, especially if the effect is genuinely perceptual (Witt, Sugovic, Tenhundfeld, \& King, 2016; Witt, in press). To make recommendations on how to proceed with research on the distance-on-hill effect, we assessed our data to determine which measure of perceived distance is most effective for capturing the effect. We compared the magnitude of the effect across various measures. In addition, we also assessed the intrasubject reliability of each measure. Intrasubject reliability can be important for ensuring replicability (through ensuring greater power), although an effect can replicate even if the measure of the effect does not have good intrasubject reliability. However, intrasubject reliability is necessary for any research involving individual differences. The use of individual differences is one potentially effective strategy for exploring the underlying mechanisms, and so assessment of intrasubject reliability can be useful for making recommendations regarding the best methodology when exploring the distance-onhills effect.

\section{Experiment 1: Visual matching}

We assessed the distance-on-hill effect in an outdoor environment using a visual matching task. If results replicate the distance-on-hill effect, this will be the first step in providing convergence to substantiate a perceptual claim. However, if the effect fails to replicate with this different measure, we would have support for the argument that these effects are judgment based. Additionally, we implemented the aforementioned illusion cancelation technique to examine whether we could cancel out the distance-on-hill effect by altering the terrain using a tarp.

\section{Method}

Participants We recruited 40 participants from the available research pool of undergraduates. One participant's data were excluded from analyses because the participant did not complete the study. All participants had normal or corrected-tonormal vision and participated as part of a requirement for an undergraduate psychology course.

Stimuli and apparatus The experiment took place on a large grassy field with a hill at one end of the field (see Fig. 1). The hill had an approximate $14^{\circ}$ slant. Distances from the participant were marked with golf tees in the ground that were not visible from where the participant was standing. A small

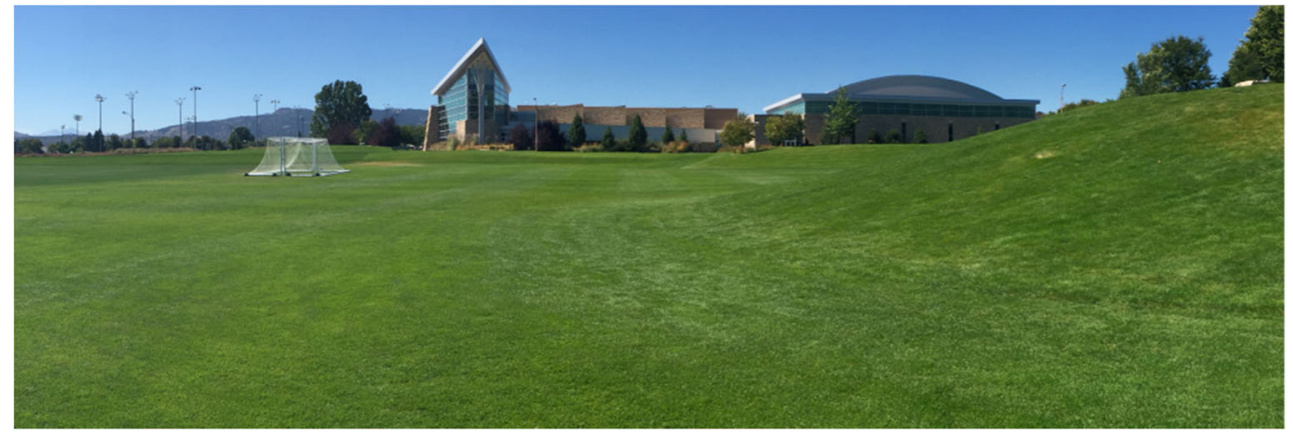

Fig. 1 A cross section of the hill used for the experiment 
circular disc cone was used to mark the target distance on each trial. A blue tarp $(3.65 \times 4.27 \mathrm{~m})$ was placed on the bottom half of the hill for participants in the tarp-present group.

Design Participants were assigned to a start condition (start near or start far) and a tarp condition (tarp present or tarp absent) in alternating order. All participants made 12 total distance estimates. The cones were placed at 6, 7,8 and 9 meters from the participant and were always placed up the hill. Each distance was presented three times, and the order of presentation was randomized. Each participant was asked to visually match the distance to the cone on the hill by instructing the experimenter to move a reference cone until the participant indicated that the distance to the reference cone was the same as the distance to the target cone. The reference cone was situated on the flat ground perpendicular to the target cone, which was presented up the hill (and thus made it hard to see both the target and reference cone without moving their head). The experimenter always stood to the left of the reference cone and moved in or out with the cone, placing it on the ground when instructed to by participants. For participants in the start-near condition, the experimenter started each trial 1.5 meters away from the participant and moved out as instructed by the participant. For the start-far group, the experimenter started 13.7 meters away and moved in toward the participant. In addition, for the group of participants assigned to the tarp present condition, the tarp was placed on the bottom of the hill. The tarp remained in the same position for the duration of the experiment. As will be discussed below, the tarp was used to examine the effects of ground texture (Sinai, Ooi, \& He, 1998) on the distance-on-hill effect.

Procedure Participants provided informed consent and were run individually. At the start, the participant stood at the base of the hill and closed her eyes ("her" is adopted as a genderneutral pronoun here). For participants in the tarp condition, the tarp was already in position prior to the start of the experiment. The experimenter placed the target cone on the hill, then stood with the reference cone at either the near $(1.5 \mathrm{~m})$ or far $(13.7 \mathrm{~m})$ starting location depending on the participant's assigned condition. The participant then opened her eyes, viewed the target cone, and directed the experimenter to move in or out until she perceived that the distance to the reference cone was the same as the distance to the target cone on the hill. Participants were told to be as accurate as possible and to make as many adjustments as necessary. The experimenter then measured the distance from the participant to the reference cone, and then started the next trial. Feedback was not given on their visual matching performance.

\section{Results and discussion}

Difference scores were calculated by subtracting the distance to the cone on the hill, from the visually matched distance on the flat ground. A positive score indicates the participant showed the expected distance-on-hill effect such that distances uphill looked farther than distances on flat ground. Difference scores were graphed in boxplots by start condition to determine outliers, and participants with difference scores beyond three times the interquartile range at one or more distances, or beyond 1.5 times the interquartile range at two or more distances were excluded. Using this criterion, four participants were deemed outliers (two had multiple difference scores that were at least 1.5 times less than the interquartile range and two had multiple differences scores that were at least 1.5 times great than the interquartile range). These participants were excluded.

Difference scores were submitted to a repeated-measures ANOVA with target distance as a within-subjects factor, and with start condition (start near vs. start far) and tarp condition (tarp present or tarp absent) as between-subjects factors. For all analyses, we report only the linear contrast for target distance. The intercept was significant, which indicates that the difference scores were significantly different from zero, $F(1,31)=14.49, p$ $<.001, \eta_{\mathrm{p}}{ }^{2}=.32$ (see Fig. 2). This result reveals the main effect of hill on the perceived distance. Targets uphill appeared farther away than targets on flat ground, which is why participants had to adjust the experimenter to be farther away. This result uses a visual matching task and replicates the previous research using verbal reports (Stefanucci et al., 2005). The main effect (linear contrast) of distance was not significant, $F(1,31)=0.24, p>$ .62 , and none of the interactions between distance and any of the other factors were significant, $F \mathrm{~s}(1,31)<1, p \mathrm{~s}>.48$, suggesting consistent effects across the target distances.

There was a significant difference between the near and far starting conditions $F(1,31)=13.27, p=.001, \eta_{\mathrm{p}}{ }^{2}=.30$ (see Fig. 3). A significant effect for whether the experimenter started near or far is consistent with past literature. Typically,

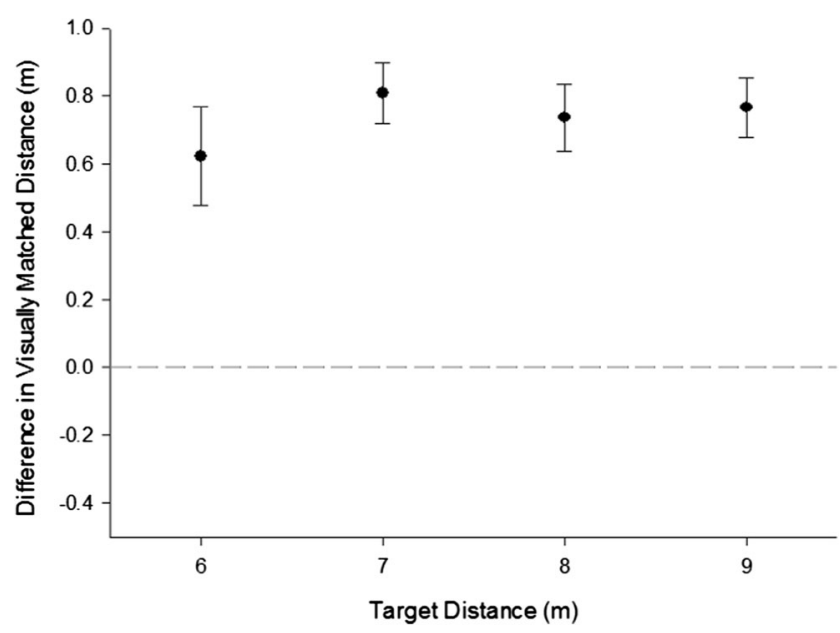

Fig. 2 Mean difference scores of visually matched distance on flat ground minus distance to the target on the hill for each target distance. The reference line is located at the point for which there would be no difference between the perceived distance on the hill and flat ground. Positive values indicate that distances on the hill were judged farther than distances on flat ground. Error bars represent 1 within-subjects SEM 


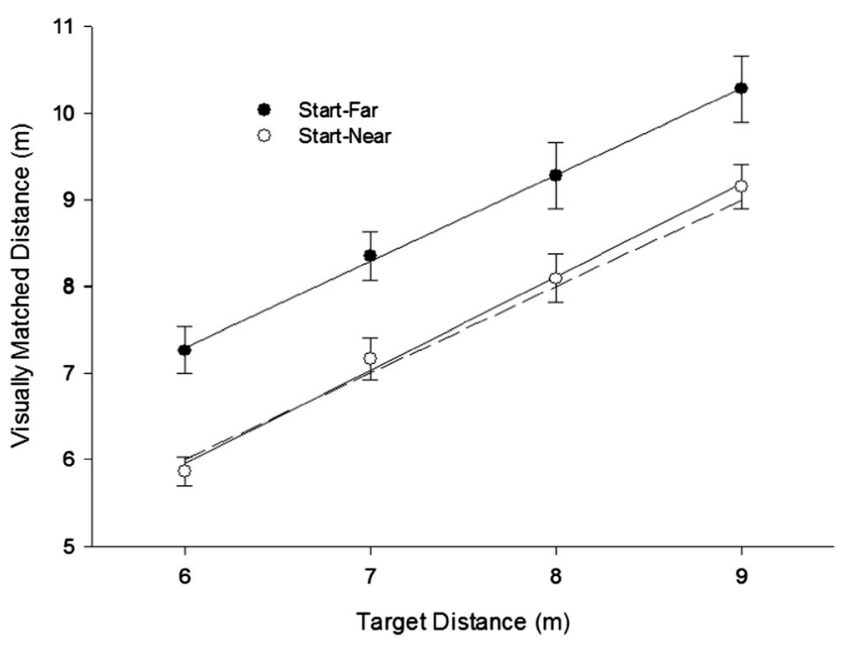

Fig. 3 Mean visually matched distance on the flat ground as a function of target distance on the hill and start condition. Dashed line indicates a perfect match. Values greater than the dashed line indicate that distance on hill was judged as farther than distance on the flat. Error bars represent 1 between-subjects $S E M$ when the starting position is close, distance tends to be underestimated compared with when the starting position is far (e.g. Witt et al., 2004). As a result, it appears that the startnear group did not show a hill effect whereas the start-far group showed a large hill effect (see Fig. 3). Statistics confirm this impression, but are not presented because they are misleading for reasons discussed below.

The difference between the two start conditions should be interpreted as a bias in the visual matching measure itself, rather than a bias in perceived distance between the two groups. This bias in the measure is because perceivers have a region wherein the reference distance would be within their just noticeable difference (JND) relative to the target distance. This is to say that there is a range of reference distances within which the participant will not be able to distinguish any difference between the target cone and the reference cone. We can think about this range as the distance between two acceptable boundaries wherein the participant would say the cone looks to be at the same distance as the target cone (see Fig. 4).
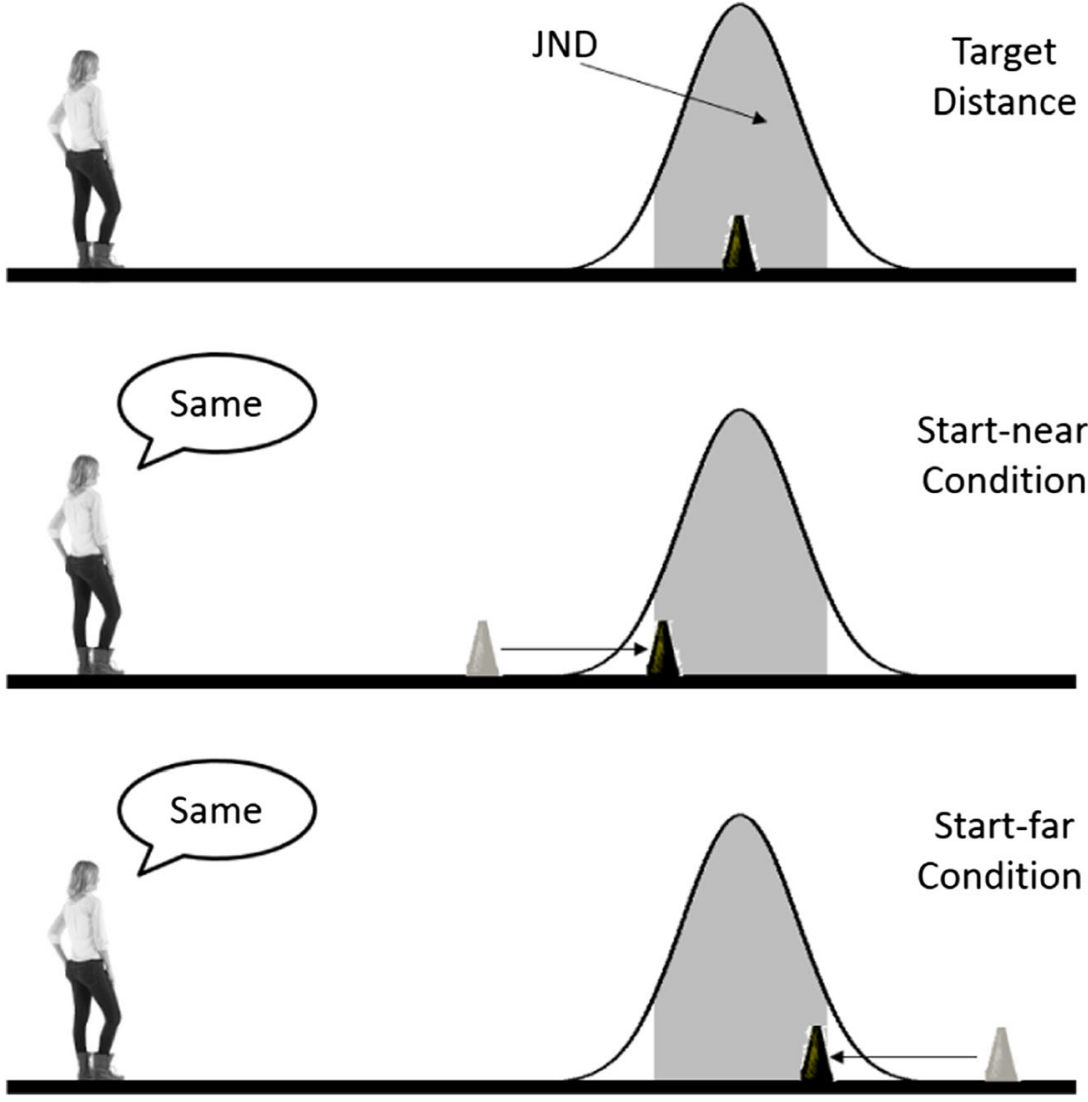

Fig. 4 Bias due to starting condition on the visual matching task. Distance between perceiver and the dark cone illustrates physical distance of the estimate. Light gray cone represents the starting location for the cone in each of the two conditions. Because of noise in the visual system, there will be a range of distances that will appear the same to the perceiver. Gray area within this curve represents this range, which is called the just-noticeable difference (JND). Any cones within this range will be perceived the same as the target distance. For participants in the start-near condition, the reference cone (starting position shown in light gray) will reach this range of acceptable distances at a closer point (middle panel) than for participants in the start-far condition (bottom panel), as shown by the final placement of the black cone. Thus, even when both groups perceive the cone to be at the same distance, their responses will differ (see difference in black cone between middle and bottom panels) 
When the reference cone is beyond the acceptable region, participants will indicate that the cone needs to be moved closer. When the reference cone is closer than the acceptable regions, participants will indicate that the cone needs to be moved farther away. However, once the reference cone is moved to within the acceptable region, participants will not make further adjustments. In the start-far condition, the reference cone will enter the acceptable region at a farther distance than for the start-near condition. While this explanation is speculative, it would elucidate why estimates are farther in the start-far condition than the start-near condition in this study and in others (e.g., Witt et al., 2004). Because of this inherent bias in the visual matching measure, researchers typically include both start conditions and take the mean estimate to obtain a more accurate measure of participants' perceptions.

The presence of a tarp did not significantly influence perceived distance, $F(1,31)=0.05, p>.83$. The tarp was used to alter the ground texture of the target distance. Sinai et al. (1998) found that distances appeared shorter when observed over differing texture gradients. We used a tarp in an attempt to replicate their findings on a hill. However, targets looked just as far away across the tarp as when no tarp was present. It is puzzling that we were not able to replicate this previous effect. In the original experiments, the ground texture varied from grass to cement. One possibility is that the texture of the tarp did not differ enough from the texture of the grass. Another possibility is that the tarp created two separate and opposite effects. Specifically, it is possible that the tarp compressed perceived distance to targets on the hill (as expected by the results of Sinai et al., 1998), and at the same time, the tarp increased in difficulty associated with walking up the hill, which served to increase perceived distance (as was shown in older adults by Sugovic \& Witt, 2013). If both effects had occurred at the same time, the two effects could have canceled each other out. Future research could attempt to tease apart these two possibilities.

There was a marginally significant interaction between the tarp and whether the experimenter started near or far, $F(1,31)$ $=3.97, p=.055, \eta_{\mathrm{p}}{ }^{2}=.11$ (see Fig. 5). Sinai et al. (1998) did not specify the starting position in their visual matching task. If they used a start position that was always near, our results with the start-near condition replicated their findings (distances looked closer when there is a discontinuity in texture gradient compared to when there texture gradient was continuous; see open circles in Fig. 5). But the results from our startfar condition did not replicate their findings and, in fact, revealed that distances appeared farther when there was a discontinuity in texture gradient compared with no discontinuity (see closed circles in Fig. 5). It could be that the tarp condition amplifies the effect of start condition by decreasing precision (or, to put it another way, increasing the JND), rather than producing its own unique effects on apparent distance. The increased variance shown for the tarp condition relative to the no-tarp condition in Fig. 5 hint at this possibility.

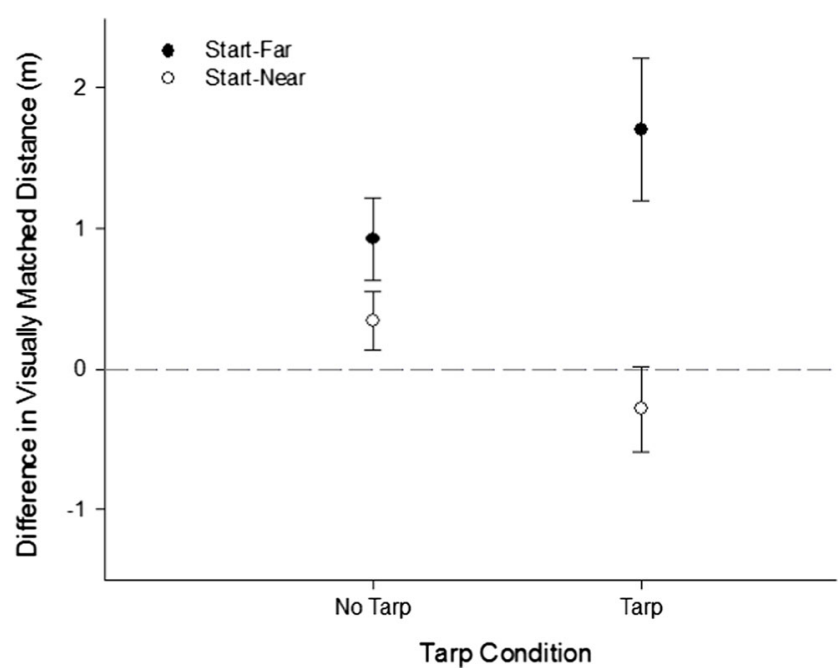

Fig. 5 Difference scores (visually matched distance minus target distance) is plotted as a function of tarp condition and experimenter starting condition. Increased difference scores indicate that distances uphill look farther than distances on flat ground. Error bars represent 1 between-subjects $S E M$

We next examined the proportion of participants who showed the distance-on-hill effect. Of the 35 participants, 22 (63\%) showed the distance-on-hill effect that targets presented uphill were judged as farther away compared with targets presented on flat ground (see Fig. 6). If we subtract out any bias due to start condition, 26 participants (74\%) show the anticipated distance-on-hill effect.

These results suggest that participants saw the distance to cones on a hill as being farther away than they appeared on the flat ground, as measured using a visual matching task. These results corroborate those found with verbal reports (Stefanucci et al., 2005). Given that verbal reports are prone to biases and

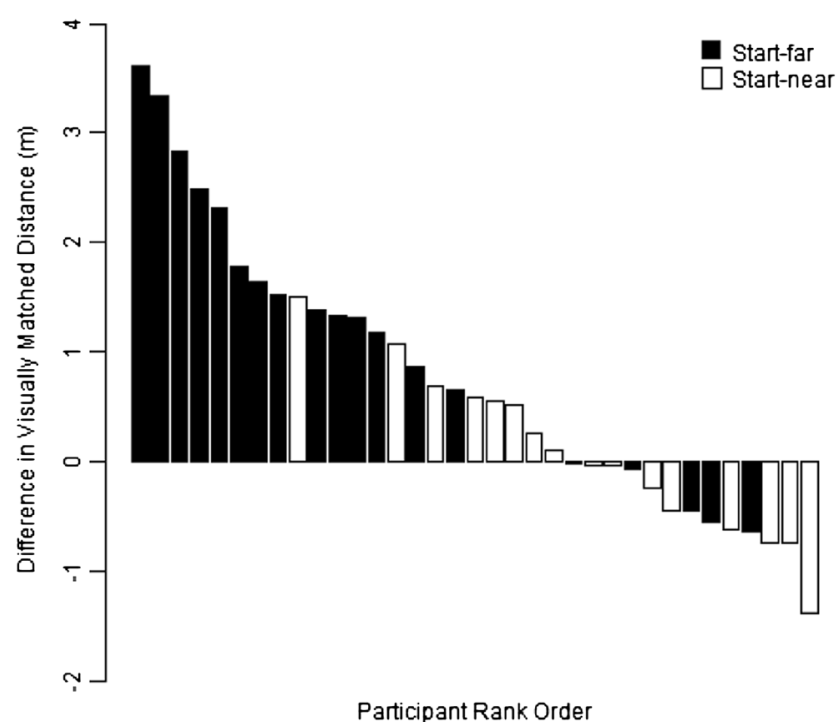

Fig. 6 Mean difference in visually matched distance on the flat ground minus the distance to the target on the hill plotted for each participant. Bar color indicates start condition. Positive values indicate the participant judged the distance on the hill as farther than the distance on the flat 
demand characteristics (Poulton, 1979), the previous results could have been due to demand characteristics rather than genuine differences in perception. By finding the same pattern with a visual matching task-for which participants simply have to adjust the reference cone until the two extents are the same - the current results demonstrate convergence across measures and support the idea that distances on hills appear farther away than distances on flat ground. We have also established this visual matching task is an effective method to assess the distance-on-hill effect.

The previous findings with verbal reports (Stefanucci et al., 2005) revealed an effect of approximately 1 meter, whereas the current results show an effect of 0.67 meters. Several factors could account for this difference. The hill in the original experiments was both steeper and longer than the hill in the current study. According to an energetics-based interpretation, increased effort to walk due to the more difficult hill should increase the size of the effect. Another option is that the increased effect size with the verbal reports could be due to a combination of a perceptual effect and a response bias effect. Given the bias inherent in the visual matching task associated with the start condition, we decided to run the same paradigm but with a blindwalking measure, which is touted for its accuracy (Loomis et al., 1992).

\section{Experiment 2: Blindwalking}

We examined the distance-on-hill effect using blindwalking as a measure of perceived distance. Blindwalking entails viewing the target distance, and then walking, with one's eyes closed, the same distance as was to the target. Blindwalking can be performed by walking directly to the target's location or walking that same distance but in a different direction. We opted for the latter option so that participants were not blindwalking uphill and so that participants were always blindwalking along the same space regardless of whether the target was presented uphill or on flat ground. Blindwalking has an advantage of other measures because it is less prone to biases and is one of the most accurate measures of perceived distance (Loomis et al., 1992). Therefore, significant results for the distanceon-hill effect using blindwalking would provide even stronger evidence consistent with a perceptual explanation. In contrast, if blindwalking does not show the distance-on-hill effect, this would be evidence against a perceptual explanation.

\section{Method}

Participants We recruited 21 participants from the available research pool of undergraduates, and with fliers posted around campus. Participants had normal or corrected-to-normal vision. One participant's data were excluded because the participant did not finish the study. Another participant's data were excluded because small children frequently ran across the hill during the experiment, causing delays and distractions.

Stimuli and apparatus We used the same hill and cones as in Experiment 1.

Design The target cone was placed 6, 7, 8 and 9 meters away from the participant on both the hill and the flat ground. The cones on the flat ground were placed at approximately 90 degrees clockwise of the hill. After each presentation of the target cone (on either the hill or flat ground), the participant turned, donned a blindfold, and blindwalked away from the hill (see Fig. 7). Participants were instructed to walk the same distance in that direction as was the distance from themselves to the target they had just viewed. Each target distance was repeated 3 times on the hill and on the flat ground for a total of 24 distance estimates. Presentation of the target cone alternated between the hill and the flat. There were four randomized orders or distances.

Procedure The experimenter had participants stand at the base of the hill and close their eyes. A cone was placed at one of the target distances $(6-9 \mathrm{~m})$ on either the hill or the flat ground adjacent to the hill. The participants were then instructed to open their eyes and look at the cone. Once they were ready, they were asked to turn $90^{\circ}$ (toward the same cardinal direction for both the hill and flat estimates), close their eyes, and walk until the distance they walked was the same as the distance that had been between themselves and the cone. Once they said they were done walking, the experimenter measured the distance from the participant to the starting area.

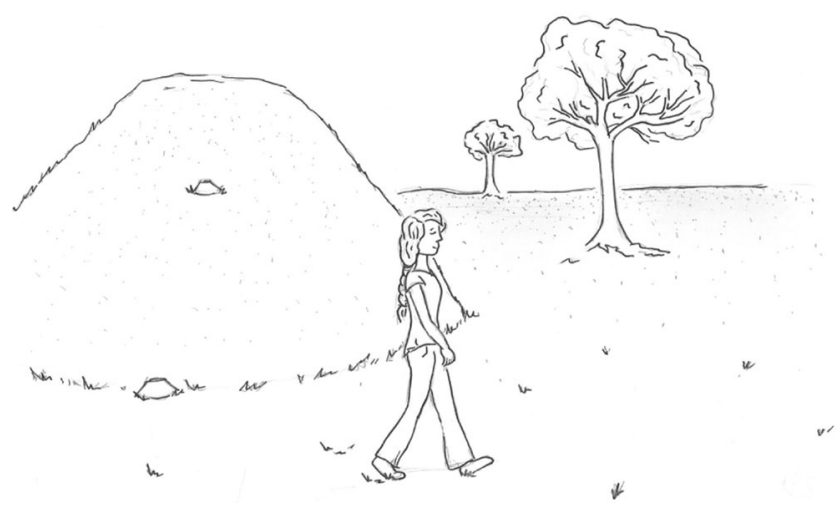

Fig. 7 The blindwalking task. Cones were placed on either the hill (as shown here) or flat ground (not shown), and participants blindwalked away from the hill to make their perceptual estimate. A cone at the base of the hill designated where the participant was to stand, and start to blindwalk 


\section{Results and discussion}

We examined the data for outliers. One participant had two blindwalked distances greater than 1.5 times the interquartile range and was excluded from further analysis. Blindwalked distance was submitted to a repeated-measures ANOVA with terrain (flat vs. hill) and target distance as within-subjects factors. Terrain significantly influenced blindwalked distance, $F(1,17)=40.40, p<.001, \eta_{\mathrm{p}}^{2}=.70$. Participants walked farther after viewing targets presented on the hill $(M=$ $7.21 \mathrm{~m}, S E=.22 \mathrm{~m}$ ) than after viewing targets presented on the flat ground $(M=6.72 \mathrm{~m}, S E=.21 \mathrm{~m})$. Target distance significantly influenced blindwalked distance, $F(7,17)=$ 231.74, $p<.001, \eta_{\mathrm{p}}{ }^{2}=.93$. Not surprisingly, participants walked farther as target distance increased. The interaction between terrain and target distance was significant, $F(1,17)$ $=5.85, p<.03, \eta_{\mathrm{p}}{ }^{2}=.26$ (see Fig. 8). Unlike in Experiment 1 , here we found that the distance-on-hill effect increased as distance increased. This increase can be seen when examining the difference scores between mean blindwalked distance when the target cone was placed on the flat ground and mean blindwalked distance when the target cone was placed on the hill (see Fig. 9). Of the 18 participants, all but 1 (94\%) showed the distance-on-hill effect such that their blindwalked distances when viewing cones on the hill were greater than their blindwalked distanced when viewing cones on the flat ground (see Fig. 10).

The finding that an action measure shows the actionspecific effect that distances presented uphill are judged as farther than when the distance is presented on flat ground may seem to contradict past results that have also used action-based measures. Bhalla and Proffitt (1999) found that verbal and visual matching tasks were biased by energetic potential in the estimation of hill slant such that hills were judged as steeper when the perceiver wore a heavy backpack

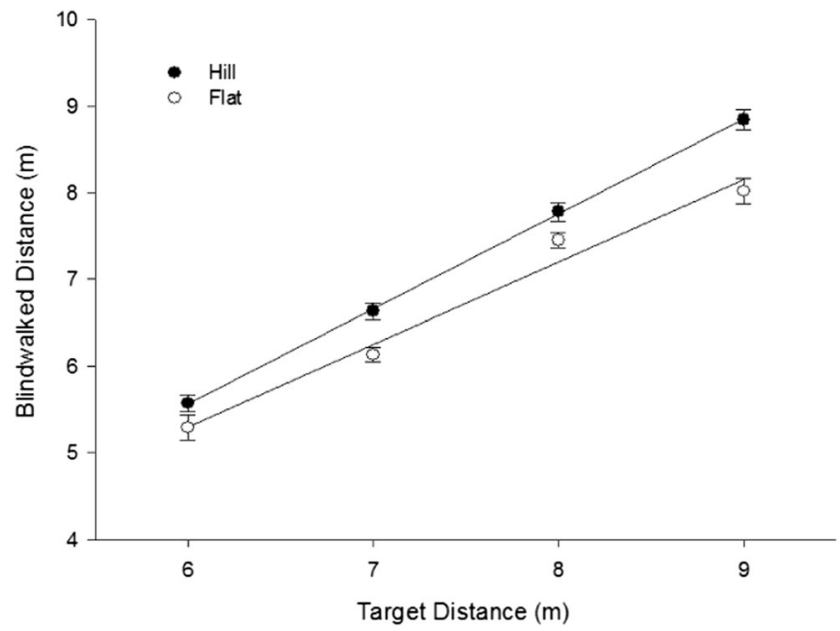

Fig. 8 Mean blindwalked distance as a function of target distance and terrain. Error bars represent 1 within-subjects SEM

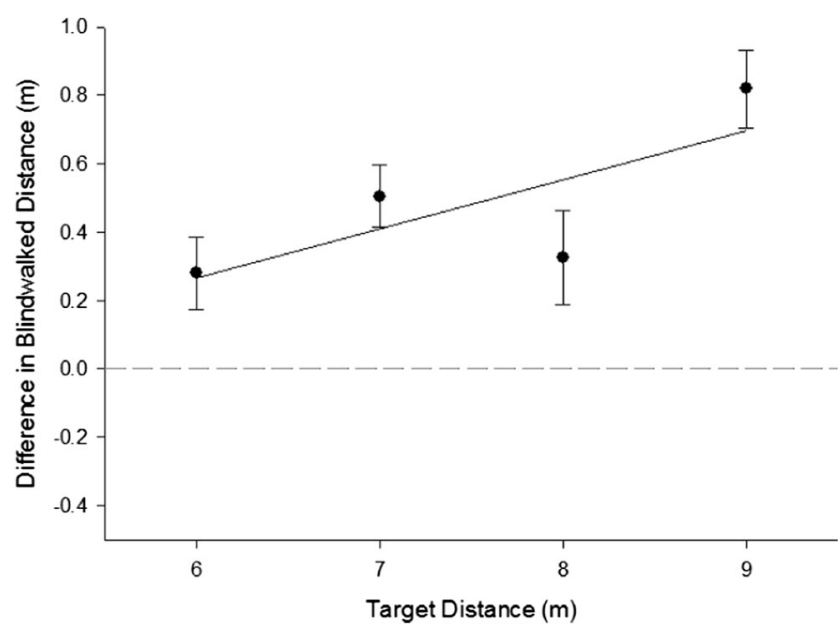

Fig. 9 Mean difference scores between the blindwalked distance of the hill and flat ground estimates, for each target distance. The reference line indicates no difference between the perceived distance on the hill and flat ground. Positive values indicate blindwalked distance was farther after viewing target on hill than on flat ground. Error bars represent 1 withinsubjects $S E M$

or was fatigued from a long run. In contrast, a haptic estimate of orienting one's hand to match the slant of the hill was not biased by energetic potential.

There are several differences that could account for the reason that blindwalking showed an effect whereas the haptic measure did not. One is that the haptic measure may be limited by biomechanics (cf. Li \& Durgin, 2012) that prevented a genuine increase in perceived slant to be measured. Another is that the haptic measure could be driven by processing in the dorsal pathway (cf. Goodale \& Milner, 1992) whereas blindwalking, which is open loop, might be driven by processing in the ventral stream, as outlined in the introduction. Other action-specific tasks have revealed effects in blindwalking (Stefanucci \& Proffitt, 2009; Witt, Proffitt, \& Epstein, 2010),

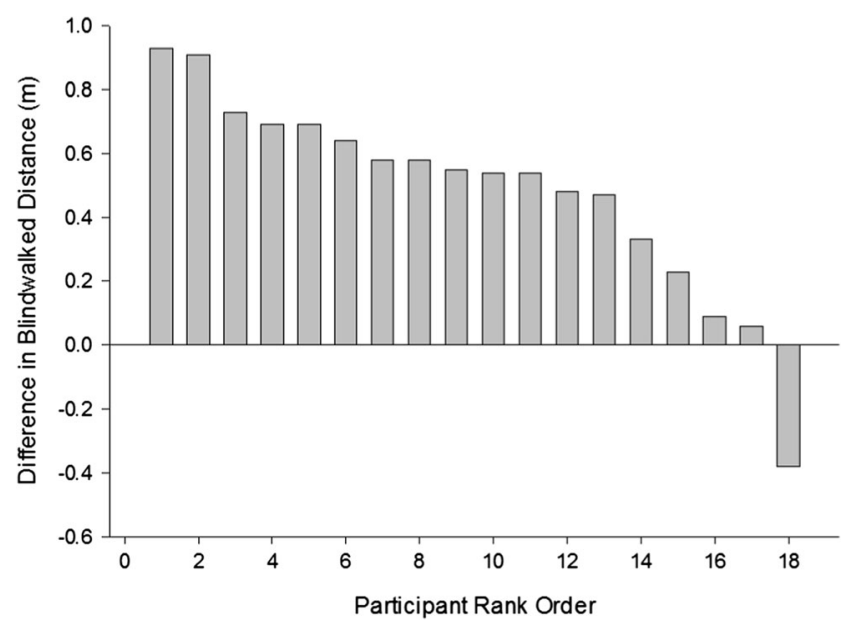

Fig. 10 Mean difference in blindwalked distance for cones viewed on the hill minus those same distances viewed on the flat for each participant. Positive values indicate blindwalked distance was farther after viewing target on hill than on flat ground 
suggesting that this measure can reveal differences in perceived distance. Had verbal estimates and visual matching both shown the distance-on-hill effect, but the measure of blindwalking had not, the data would have cast doubt on a perceptual interpretation of the distance-on-hill effect.

The blindwalking task used here had participants blindwalk in a different direction than that of the target. This version of the blindwalking task had participants blindwalk along the same ground regardless of whether they had viewed the target on the hill or the target on the flat ground. This helps ensure that there were not biases in the response itself that could account for the results. A previous study found similar performance when using this variation of the blindwalking task as when participants are instructed to blindwalk to the target itself (Sinai et al., 1998).

Once again, the results are consistent with the idea that participants saw the cones as being farther away when they were on the hill than when they were on the flat ground. The results demonstrate that blindwalking is another effective method for obtaining the distance-on-hill effect. These results further substantiate the idea that the effects are perceptual in nature by demonstrating convergence across verbal (Stefanucci et al., 2005), visual matching (Experiment 1), and blindwalking tasks (Experiment 2).

\section{Experiment 3: Multiple measures}

Having established that both visual matching and blindwalking are effective methods of data collection within the paradigm, and providing initial evidence for convergence, we next compared estimates from one measure to another. By running all three methods within the same group of participants, we can compare and contrast these techniques for assessing perceived distance. This also provides an additional way to assess convergence, by looking within an experiment instead of across experiments, and provides an opportunity to attempt to replicate the findings from the first two experiments.

\section{Method}

Participants We recruited 25 participants from the available research participant pool. One participant's data were excluded because the study had to be stopped early due to grass mowing that was taking place during the experiment.

Stimuli The hill and cones were the same as used in the first two experiments.

Design Participants completed three blocks of trials. In each block, a different measure of perceived distance was used. These measures were verbal estimates, visual matching, and blindwalking. The order was counterbalanced across participants. Presentation always alternated between flat and the hill, but order of distance was randomized. The targets were placed at 7,8 , and 9 meters away in all three blocks.

Procedure After providing consent, participants were walked to the bottom of the hill and told to straddle a cone placed at the base. They were then told that they would be estimating distances to cones placed on the hill and on the flat ground in a variety of different ways, each of which would be explained immediately before being asked to do so.

For the verbal estimates, participants were instructed to verbally estimate the distance to the cone. They were not provided with a measurement metric (i.e. feet, meters, etc.), and if they inquired, were told to use whichever metric with which they felt most comfortable. One practice trial was given for both the flat and the hill at either 5 or 6 meters, but participants were not told it was a practice trial. Participants then completed 6 test trials, alternating between the hill and flat, such that they estimated the distance to the 7,8 , and 9 meter distances for both.

The visual matching was conducted in the same way as in Experiment 1. Participants were told to move the experimenter in or out until the distance between themselves (the participant) and the reference cone that the experimenter was moving, was the same as the distance between themselves and the target cone on the hill, which was also placed at 7,8 or 9 meters. Participants were given one practice trial at either 5 or 6 meters, which they were not told was practice, and matched each of the experimental distances just once, for a total of one practice trial and three test trials. They were told to be as accurate as possible and they were allowed to ask the experimenter to put down and then pick up the cone again if further adjustments were needed. Once the participant said the two distances matched, the experimenter measured the distance between the reference cone and the participant. This was done between each trial, and participants were not given feedback. For one group of participants, the experimenter always started near ( $2 \mathrm{~m}$ away), and for the other group, the experimenter always started far (14 $\mathrm{m}$ away).

The blindwalking procedure was the same as in Experiment 2, with the exception that participants were instructed to practice on their own by picking a spot on the ground and attempting to walk to it until they felt comfortable with the blindwalking task. Participants were then given two practice trials at either 5 or 6 meters, with one on the hill and one on the flat (for instance, if they were shown the 6-meter cone on the hill, they would then be shown the 5 meter on the flat). Once again, participants were not told these were practice trials. They then completed the six experimental trials (three target distances at 7, 8, and 9 meters, and a hill and a flat presentation each). The target cone alternated being placed 
on the hill and flat ground, and the target cone was set to 7,8 , and 9 meter distances for both. The experimenter measured the distance walked between each trial.

\section{Results and discussion}

We computed difference scores for each measure for each distance for each participant. For the verbal estimate and blindwalking task, difference scores were computed by subtracting the flat estimate from the hill estimate. For the visual matching, difference scores were calculated by subtracting the actual distance of the target cone that was on the hill from the distance to the final position of the reference cone on the flat ground. Despite these differences in calculations, all three difference scores provide a relative measure of estimated distance to targets presented on a hill versus flat ground. Using the same criteria as in the first two experiments, no one was identified as an outlier. However, one participant produced vastly different responses on the visual matching task to the 8-meter target than to the other targets (less than $4 \mathrm{~m}$ for the 8-m target and greater than $7 \mathrm{~m}$ for both 7 - and $9-\mathrm{m}$ targets), and was therefore excluded.

We conducted a repeated-measures ANOVA with difference score as the dependent measure and measure type and target distance as within-subjects factors. The intercept was significantly greater than zero, $F(1,22)=28.86, p<.001$, $\eta_{\mathrm{p}}{ }^{2}=.57$. This reveals the distance-on-hill effect as shown in the previous experiments.

Next, we examined whether the magnitude of the distanceon-hill effect varied across the three measures. Measure type did not significantly influence the difference scores, $F(2,44)=$ $0.20, p>.82, \eta_{\mathrm{p}}{ }^{2}<.01$ (see Fig. 11). This demonstrates clear convergence across three unique types of measures in showing that targets on hills are judged as farther than targets on flat ground regardless of the measure used. This convergence is

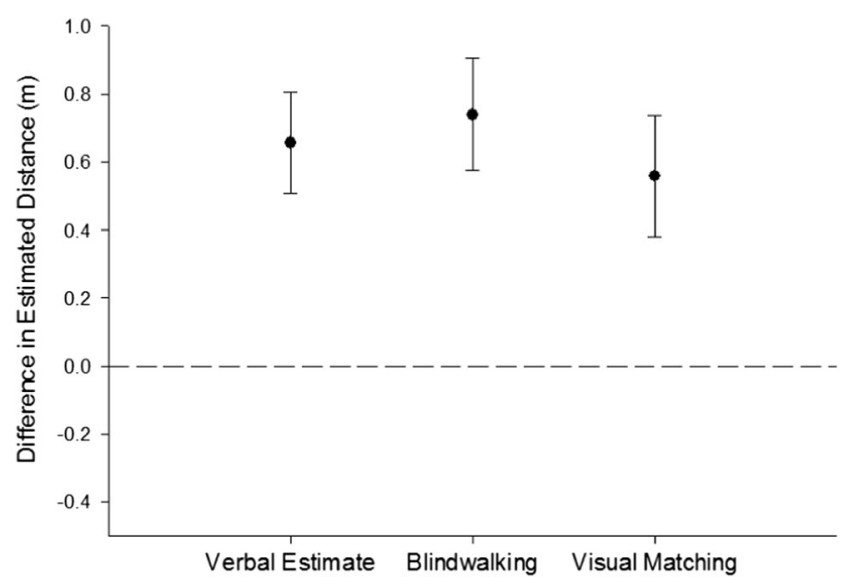

Fig. 11 Mean difference in estimated distance for each of the perceptual measures. A positive score indicates that the distances on the hill were reported as being farther away than the distances on the flat ground. Error bars represent 1 within-subjects SEM evidence supporting the idea that the underlying effect is perceptual (e.g., Foley, 1977; Gogel, 1990). To further substantiate this convergence, we also conducted separated repeatedmeasures ANOVAs for each measurement type, which is discussed below.

The linear effect for distance was significant, $F(1,22)=$ $4.33, p=.05, \eta_{\mathrm{p}}{ }^{2}=.16$. The difference between estimates for targets on the hill versus flat increased as distance increased. The interaction between measurement type and distance was not statistically significant, although there was a slight trend, $F(1,22)=3.14, p=.09, \eta_{\mathrm{p}}{ }^{2}=.13$ (see Fig. 12). We explored the data for each condition. Although this means the overall picture is less clear, it provides better transparency of the data.

We conducted separate repeated-measures ANOVAs for each measure type. For each ANOVA, difference score was the dependent factor and distance was a within-subjects factor. For visual matching, the intercept was significant, $F(1,22)=$ 4.83, $p=.04, \eta_{\mathrm{p}}{ }^{2}=.18$, although the statistical significance was more marginal compared with the other measures. This is likely due to the increased variability due to start condition. When we included start condition as a between-subjects factor, the significance decreased and the effect size increased, $F(1,21)=7.30, p=.01, \eta_{\mathrm{p}}^{2}=.26$. The linear contrast for distance was significant, $F(1,21)=4.62, p=.04, \eta_{\mathrm{p}}^{2}=.18$, showing a modest increase in the distance-on-hill effect as distance increased. For blindwalking, the intercept was significant, $F(1,22)=17.71, p<.001, \eta_{\mathrm{p}}^{2}=.45$. The linear contrast for distance was marginally significant, $F(1,22)=3.74, p=$ $.07, \eta_{\mathrm{p}}{ }^{2}=.15$. Although the blindwalking measure showed the largest increase in the distance-on-hill effect as distance increased, this increase was only marginally significant. For verbal estimates, the intercept was significant, $F(1,22)=$

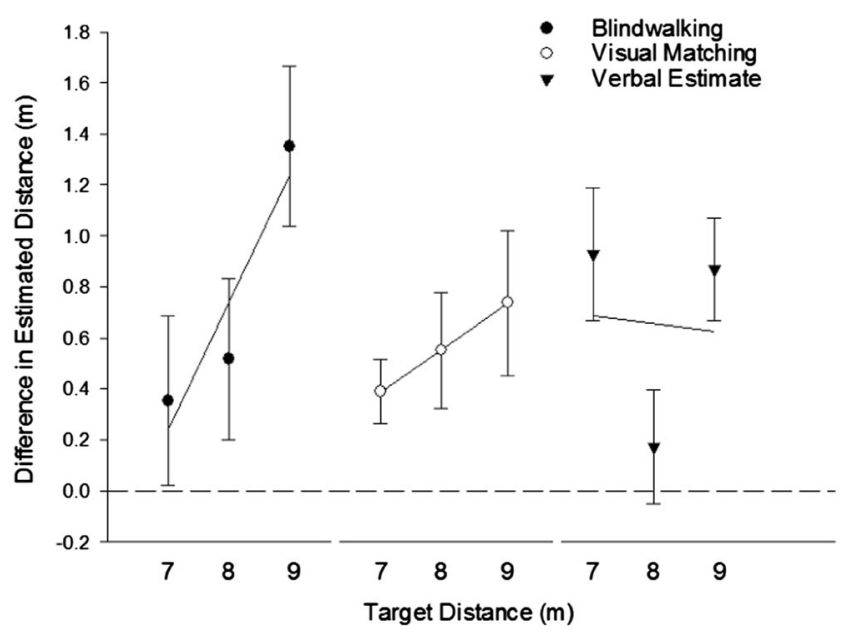

Fig. 12 Mean difference in estimated distance for each of the perceptual measures as a function of target distance. Lines represent linear regressions. Dotted line indicates score for when targets on the hill are estimated to be the same as on flat ground. A positive score indicates that the distances on the hill were reported as being farther away than the distances on the flat ground. Error bars represent 1 within-subjects SEM 
$14.36, p=.001, \eta_{\mathrm{p}}{ }^{2}=.40$. The linear contrast for distance was not significant, $F(1,22)=0.07, p>.78, \eta_{\mathrm{p}}{ }^{2}<.01$.

It should be noted that all three measures showed the distance-on-hill effect, as indicated by the significance of the intercept in each case. They also all converge on similar values. This aspect of the data will be discussed further in the Methodological Considerations section.

The data reveal that the measures are not consistent with respect to whether or not the distance-on-hill effect differs across distances. The visual matching task showed no change in the distance-on-hill-effect as distance increased in Experiment 1, but did so in Experiment 3. Blindwalking showed significant increases in both Experiments 2 and 3. Verbal estimates did not show an increase in Experiment 3. Thus, there are some discrepancies in the data. From a theoretical perspective, the action-specific account should predict an increase in effects as distance increases because farther distances demand more effort. However, it may be difficult to assess this given a number of factors. First, the distance-onhill effect should only increase to the extent that the difference in the energetic demands also increase. An experiment designed to assess this particular prediction should use a wider range of distances than what we used in the current experiments. Second, the measure used to assess perceived distance should be sufficiently sensitive such that an effect is not masked by variance. According to Weber's law, as distance increases, variance also increases. In addition, for verbal estimates and visual matching (but not blindwalking), there is compression such that these measures show reduced sensitivity to distance as distance increases. This compression might also mask any increases that might have otherwise been found in the distance-on-hill effect as distance increases. To fully investigate this aspect of the distance-on-hill effect, we recommend additional research with a wider range of distances and more trials.

\section{Experiment 4: Beliefs about the distance-on-hill task}

Experiments 1-3 explored the perceptual nature of the distance-on-hills effect by using strategies related to visual comparison tasks, action-based tasks, and convergence across measures. However, other strategies have also been offered to explore this critical question. One is to survey participants after the experiment to assess if anyone was able to infer the study's purpose. While we did not have the foresight to include a postexperiment questionnaire in the above studies, we wanted to explore how 'predictable' these results are. We conducted a survey in which the experimental set up from the studies here was described, and participants indicated the expected effect on estimated distance.

\section{Method}

Participants We recruited 47 participants from the available research participant pool.

Stimuli Participants were administered the survey on a computer through E-Prime.

Design Participants were shown the image in Fig. 13 and presented with the following question: "Jane is standing at the base of a hill looking at a cone on both the hill and the flat ground behind her. Both cones are 10 meters away from her feet. Which of the following best describes how Jane perceives them?" Participants were given three answer choices, which were presented in random order: "The cone on the hill looks closer than the cone on the flat ground because the cone on the hill is closer to her eyes" (closer); "The cone on the hill looks further away than the cone on the flat ground because walking to the cone on the hill would take more energy" (farther); "The cone on the hill looks the exact same as the cone on the flat ground, because they are in fact the same distance away from her" (same). Participants were then presented with a fourth option that was always the final one that read "The cone on the hill looks when compared to the cone on the flat because ," where the participants were invited to fill in the blanks if they felt the answer was not available in the first three choices (other).

Procedure The survey was conducted as part of a filler task during an unrelated memory experiment. The survey also contained one other question about a different actionspecific effect, in counterbalanced order with the question about the hill.

\section{Results and discussion}

Of the 47 participants, only $14(29.78 \%)$ correctly chose the farther response. In contrast, 30 participants $(63.8 \%)$ chose the opposite direction of the effect (the closer response). One participant selected "other" and wrote "closer [because] it is perceived at an angle." The remaining three participants (6\%) selected the same response. The proportion of those that said the cone on the hill would look closer was significantly different from those who said it would look farther away; $z=$

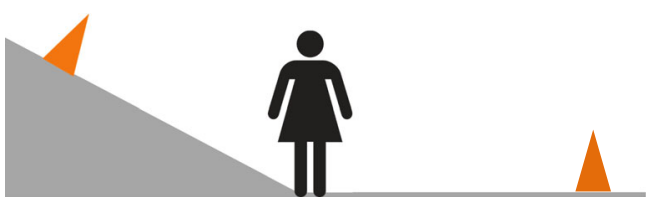

Fig. 13 Image shown to participants in tandem with the question about the direction of the distance-on-hill effect 
$3.31, p<.001$. In contrast to a response bias explanation, participants were inaccurate at inferring the expected effect.

If participants adjust their responses based on their inference of the experiment's purpose, this pattern suggests that measures of the distance-on-hill effect actually underestimate the magnitude of the effect because they do not take into account response bias to respond that targets on hills are actually closer. Given that only a small portion of participants could accurately guess the anticipated effect, and that over twice as many guessed the opposite effect, this effectively rules out a response bias explanation and renders the need to include cover stories or postexperiment surveys obsolete for this particular action-specific task.

\section{Methodological considerations}

Having established that the distance-on-hill effect emerges using multiple types of perceptual measures, and therefore providing convergence, the data are consistent with a perceptual explanation of the distance-on-hill effect (cf. Philbeck \& Loomis, 1997). Had one or multiple measures not shown the effect, the data would have cast doubt on the idea that targets presented up a hill appear farther away compared to targets presented on flat ground. In addition to these theoretical considerations, the data also speak to a number of methodological considerations that could be useful for future studies.

\section{Magnitude of the effects}

Table 1 shows the mean difference score (and SEM), effect size, and number of participants showing the distance-on-hill effect across all of the experiments. The mean difference score is fairly similar across all measures. Note also that running three blocks of trials with the same measure (as was done in Experiments 1 and 2) did not substantially alter the mean difference score relative to running three blocks of trials with different measures (Experiment 3). However, this procedural difference did impact the mean $S E M$, especially for blindwalking.
The effect sizes suggest that the blindwalking measure yields the largest effect of the three measures, both in the direct comparisons of the measures within Experiment 3 and between experiments. Given the notable accuracy of blindwalking, the pattern that the blindwalking measure produced such strong effect sizes is especially compelling evidence for the claim that the distance-on-hill effect is perceptual. With respect to which measure to implement in future research, neither mean difference score nor number of participants showing the effect favor one measure over another. Based on effect size, there seems to be some advantage for blindwalking.

\section{Reliability}

Based purely on magnitude of the effect, we do not have a strong recommendation for one measure over another. This recommendation changes when we consider the intrasubject reliability of the three measures. The data show that each of these measures possess group-level reliability, which is to say that the effect reliably replicates in each experiment. However, we also want to consider individual, or intrasubject, reliability. A measure can have high group reliability (i.e., consistently replicates from experiment to experiment) but have low intrasubject reliability (i.e., an individual participant's score on some trials is not predictive of that person's score on other trials). The Stroop effect is one such example of an effect that has high group reliability and poor intrasubject reliability (e.g., Franzen, Tishelman, Sharp, \& Friedman, 1987; Strauss, Allen, Jorgensen, \& Cramer, 2005). With respect to group effects, intrasubject reliability is not necessarily required. But for researchers interested in effects at the individual level, high intrasubject reliability is necessary to pinpoint a given participant's distance-on-hill score.

Individual reliability is important for several reasons. First, higher reliability yields an increase in power (Cohen, 2013). At a time wherein underpowered studies have led to effects that are nonreplicable, paradigms that allow for greater overall power with the same number of participants should be sought out (Open Science Collaboration, 2015). Given that there have been reported issues of failures to replicate action-specific effects

Table 1 Comparison of the magnitude of the effect for Experiments 1-3

\begin{tabular}{lllllll}
\hline Experiment & Measurement type & $N$ & Mean difference score $(\mathrm{m})$ & Mean SEM $(\mathrm{m})^{\mathrm{a}}$ & $\eta_{\mathrm{p}}^{2}$ & Participants showing effect (\%) \\
\hline 1 & Visual match & 35 & .67 & .11 & .32 & 62.9 \\
2 & Blindwalk & 18 & .48 & .11 & .70 & 94.0 \\
3 & Verbal estimate & 23 & .68 & .23 & .40 & 82.6 \\
3 & Visual match & 23 & .56 & .21 & .26 & 69.6 \\
3 & Blindwalk & 23 & .74 & .32 & .45 & 78.3 \\
\hline
\end{tabular}

${ }^{a}$ Calculated within-subjects for each experiment 
(e.g., Woods et al., 2009), increased power is especially important for interpreting significant effects as well as failures to replicate. In the case of the distance-on-hill effect, the reported $p$ values have always been highly significant (all $p \mathrm{~s}<.007$, including Experiments 1-3 and Experiments 1-2 in Stefanucci et al., 2005). When a distribution of p-values is skewed towards such low values, this is "diagnostic of evidential value" as opposed to $p$ hacking, low power, or other issues related to publication bias (Simonsohn, Nelson, \& Simmons, 2014, p. 535).

Secondly, high intrasubject reliability allows researchers to better understand the underlying mechanism by exploring various factors that impact the distance-on-hill effect. One way to do this is to leverage individual differences. Examining whether some individuals are more or less prone to action-specific biases could provide important insights into how action exerts its influence on spatial perception. For example, individuals with autism spectrum disorders have impaired ability to perceive affordances (Linkenauger, Lerner, Ramenzoni, \& Proffitt, 2012). If these individuals also show reduced actionspecific effects such as the distance-on-hill effect, this could speak to a role for affordance perception in action-specific effects. As far as we know, this is the first time that intrasubject reliability has been explored relative to an action-specific effect.

In order to compute the intrasubject reliability, we computed the mean difference score across the 6- and 9-meter distances, and the mean difference score across the 7- and 8meter distances for Experiments 1 and 2. For Experiment 3, we computed the mean difference score across the 7- and 9meter distances to compare to the difference score at 8 meters. We then correlated the extreme values (i.e., the mean of 6 and $9 \mathrm{~m}$ for Experiments 1 and 2, or the mean of 7 and $9 \mathrm{~m}$ for Experiment 3 ) with the middle values (i.e., mean of 7 and $8 \mathrm{~m}$ for Experiments 1 and 2 or $8 \mathrm{~m}$ for Experiment 3). Because we essentially split our data in half, we calculated reliability with the Spearman-Brown prophecy formula (MacLennan, 1993). The reliability score indicates how predictive one estimate is for the other estimate within the same participant. SpearmanBrown prophecy coefficients greater than .80 are considered to have adequate reliability (and have been bolded in Table 2),

Table 2 Reliabilities for Experiments 1-3 when comparing difference scores (hill estimates minus flat) for extreme (closest and farthest) distances to middle distance(s)

\begin{tabular}{lllll}
\hline Experiment & Measurement type & $N$ & $\rho^{*}{ }_{\mathrm{xx}}$ & Correlations \\
\hline 1 & Visual match & 35 & $\mathbf{. 9 4}$ & $\mathbf{. 9 0 * * *}$ \\
2 & Blindwalk & 18 & .37 & .23 \\
3 & Verbal & 23 & .13 & .07 \\
3 & Visual match & 23 & $\mathbf{. 8 6}$ &. $\mathbf{7 6 * * *}$ \\
3 & Blindwalk & 23 & -.08 & -.04 \\
\hline
\end{tabular}

Note. $\rho^{*}{ }_{\mathrm{xx}}$, is the Spearman-Brown prophecy coefficient. $* * * p<.001$ Bold signify the only measure with high reliability and coefficients greater than .90 are considered to have good reliability. A cut-off as low as .60 is sometimes used for exploratory research. As shown in Table 2, only the visual matching provides a reliable measure.

High reliability indicates that the obtained scores adequately capture some component of an individual's perceptual experience, presumably their distance-on-hill effect. The data cannot speak to whether distance-on-hill effects are stable across time because we only measured the effects within a single session. However, the data show that researchers could explore the stability of these effects as long as the researcher uses the visual matching measure.

The low reliability scores for verbal estimates and blindwalking do not mean that these measures cannot capture the distance-on-hill effect at the group level. As shown in the reported experiments, these measures are adequate to do just that. Instead, what the lack of reliabilities show is that these measures cannot pinpoint an individual's susceptibility to the distance-onhill effect. While the blindwalking measure yielded the largest effect size, studies looking to examine individual differences should use the more reliable measure of visual matching.

There are a couple reasons that this visual matching measure is likely to be the most reliable. Given the significant effects of starting condition (start near vs. start far), there may be certain inherent binding aspects that promote the clustering of a participant's scores. This could lead to an increase in reliability, if the estimates are localized at the edges of the $\mathrm{JND}$, as was discussed previously. If this were the case, we would expect to see a clear delineation between the estimates for the start-near versus start-far group, however that does not seem to be the case when looking at the data (see Fig. 14).

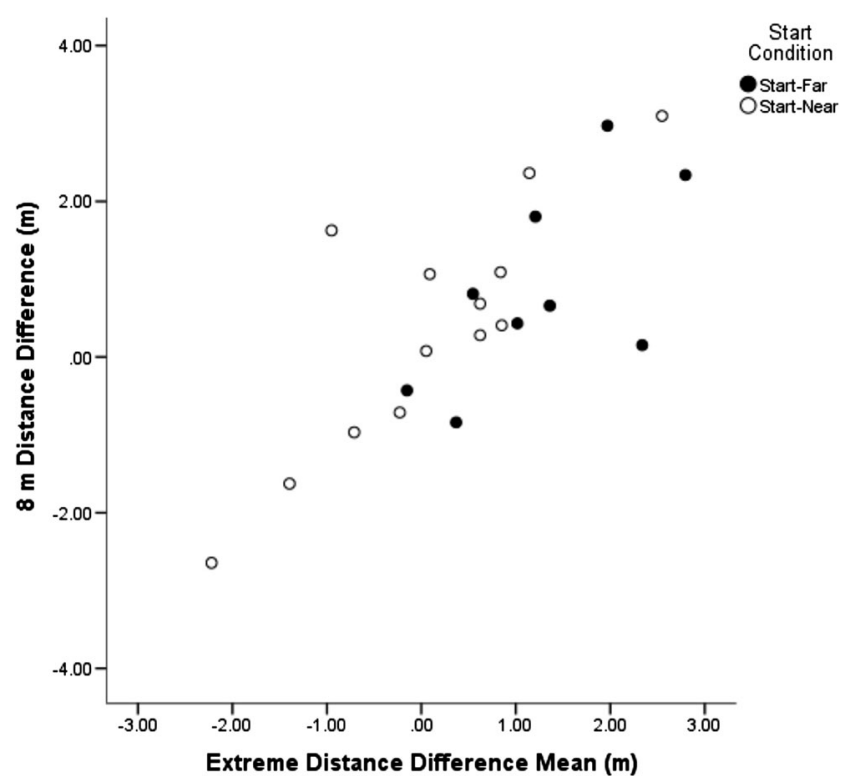

Fig. 14 Visual matching difference score at 8 meters plotted as a function of the mean visual matching difference score at 7 and 9 meters and as a function of start condition. Each point represents the data from one participant from Experiment 3 
The more likely reason why visual matching, but not verbal estimates or blindwalking, is reliable relates to how the difference scores were calculated. For visual matching, the difference score was the difference between an estimated extent and a physical extent. Thus, the calculation involved only one value contained variability. For verbal estimates and blindwalking, the difference score was the difference between two estimated extents, so both values contained variability. Difference scores tend to be unreliable (e.g., Richler, Floyd, \& Gauthier, 2014). For instance, when analyzing the difference scores related to the Stroop task, reliability was low, but when analyzing response latencies within a condition (rather than across conditions), reliability was higher (Strauss et al., 2005). Although the visual matching is technically a difference score, it is not a difference score in the same sense as for verbal estimates, blindwalking, or comparison of conditions like in the Stroop task because the visual matching difference score is the difference between an estimate and a physical value. Note that the meaning of the difference score for the visual matching is still the same as the meaning of the difference scores for verbal estimates and blindwalking because all three provide an estimate of the relative difference between perceived distance to targets on a hill to targets on a flat ground. Thus, the difference scores can be directly compared to one another.

This good reliability of the visual matching task could prove to be beneficial to the field moving forward in efforts to explain individual differences on action-specific effects, to examine the nature of action-specific perception, and to explore longitudinal changes over time of action-specific perception.

\section{General discussion}

The data are consistent with the claim that distances on hills appear farther than they do on the flat ground. Distances that require more effort to traverse due to being up a hill appear longer compared with distances that require less effort to traverse. This effect of effort on perceptual judgments suggests that perception is influenced by a person's ability to act. These and similar effects have been called action-specific effects on perception (Witt, 2011).

However, critics of this claim argued that the observed effect is not due to differences in perception and are instead driven by response biases (Durgin et al., 2009; Firestone \& Scholl, 2016). A response bias is a bias to change the estimate of perception even though perception itself is not different. The previous research documenting the distance-on-hill effect used verbal estimates to assess perceived distance (Stefanucci et al., 2005), and verbal estimates are prone to response biases, demand characteristics, and other biases (Pagano \& Isenhower, 2008; Poulton, 1979; Stevens, 1957). In other words, participants may have reported that the targets on the hill were farther than the targets on the flat even if the hill targets did not look farther because of task demands or experimenter effects.

Differentiating perceptual effects from judgment-based effects is an important but difficult problem. It is important because a comprehensive account of vision requires understanding the factors that influence perception. It is difficult because researchers can only measure behaviors and judgments, and from these, make inferences about the underlying perception. Using several strategies, the current data support a perceptual interpretation of the distance-on-hill effect.

The distance-on-hill effect was found using a visual matching task, which has the advantage that perceivers simply have to visually compare two distances without the challenge of having to assign numbers to the extents (as in verbal reports). The distance-on-hill effect was found using a blindwalking task, which has the advantage of being an action-based measure. Action-based measures are not as likely to be prone to certain biases, such as the possibility of multiple interpretations (cf. Wood et al., 2009).

In addition, convergence was found across verbal estimates, visual matching, and blindwalking tasks. Exploration of convergence was a strategy promoted by the field of research on distance perception is to look for convergence across a range of measures (Foley, 1977; Gogel, 1990; Loomis \& Philbeck, 1997). If a similar pattern of responses emerges across all measures used, this would be consistent with the claim that the effect is truly perceptual. However, if the effect emerges only with, for example, the verbal estimates that have been used in the past, and not in visual matching or action-based measures, this would substantiate the claim that the studied effect is likely postperceptual or judgment based.

Although convergence was found with respect to the main distance-on-hill effect, convergence was not found with respect to whether the distance-on-hill effect increases as distance increases. Thus, the data are insufficient to make the claim that the distance-on-hill effect changes with target distance. Given that the experiments were not designed to assess this aspect of the task, it would be premature to draw strong conclusions at this stage. Future studies should assess this potential relationship given that it should be predicted by an action-specific account of perception. However, as discussed in Experiment 3, there are several methodological issues that would need to be addressed including using a wider range of distances.

Another strategy was to assess participants' predictions of the distance-on-hill effect. Participants were given a description of the experiment and asked to predict the subsequent effect on perceived distance. Less than a third predicted that distances uphill would appear farther. The majority actually predicted the opposite effect. These results are damning to a response bias account that depends on participants having the ability to discern the purpose of the experiment. 
A limitation is that this paradigm involves two conditions that differ not just in the energetic requirements to act but also in the visual differences as well. This is an important concern because even if the effects are perceptual, they may not be evidence for an effect of action on perception but rather due to the visual differences instead (Firestone \& Scholl, 2016). Stefanucci and colleagues (2005) originally argued that the visual differences should lead to the opposite predictions given that people see hills as steeper than the hill's actual slant (cf. Proffitt, Bhalla, Gossweiler, \& Midgett, 1995), and thus the distance to the targets on hills should appear closer (see Fig. 15). However, one could also argue that targets on hills should appear farther away because the target is located closer to the horizon, and height in visual field provides an optical cue for an object's distance (Cutting \& Vishton, 1995). It is unclear to us how these various possibilities could be differentiated. One thought is to adjust the slant of the hill, which should impact perceived distance according to an actionspecific account. But it would also impact perceived distance according to the height in visual field account and thus would be uninformative regarding which factor is responsible for these effects. It is also unknown how height in visual field is informative in the context of a slanted surface, making it especially challenging to make predictions for the two accounts. This is an important problem that will require resolution.

Finally, the role of intention to act needs to be further explored to fully understand these effects as they relate to intention. Past research has suggested that action capabilities only affect perception when the perceiver intends to act (Witt, Proffitt, \& Epstein, 2004, 2005), but several studies show action-specific effects even when there is no requirement to act, such as in the current studies. Moreover, some research has even shown action-specific effects when viewing lifesized projections of images for which action would not even be possible (Taylor-Covill \& Eves, 2013). One possibility is that in the context of a target placed on the ground, there is an implicit intention to walk to that target even if not explicitly instructed to do so. Furthermore, life-sized projections of images are likely to involve many of the same perceptual processes as when viewing a real scene, thus permitting processes involved in anticipating action (such as predictive forward models) even though these actions are not possible. Indeed, many studies have shown action-related effects such as priming of actions just when viewing images (Guettling, Park, Kenemans, \& Neggers, 2013; Tucker \& Ellis, 1998; Witt, Kemmerer, Linkenauger, \& Culham, 2010; Yang \& Beilock, 2011). These issues are important but would be difficult to resolve given the complexities in measuring a person's intention.

Another advancement of the current experiments relates to intrasubject reliability of this action-specific effect. In terms of the magnitude of the effect, all three perceptual measures produced similar effect sizes, with a slight advantage for blindwalking. However, only the visual matching task was reliable at the intrasubject level. The reason that the visual matching task, but not the verbal estimates or blindwalking tasks, were reliable may relate to how the calculation of the difference scores involved only one measure with variability, as opposed to two measures. All other action-specific measures to date involve the comparison of estimates across

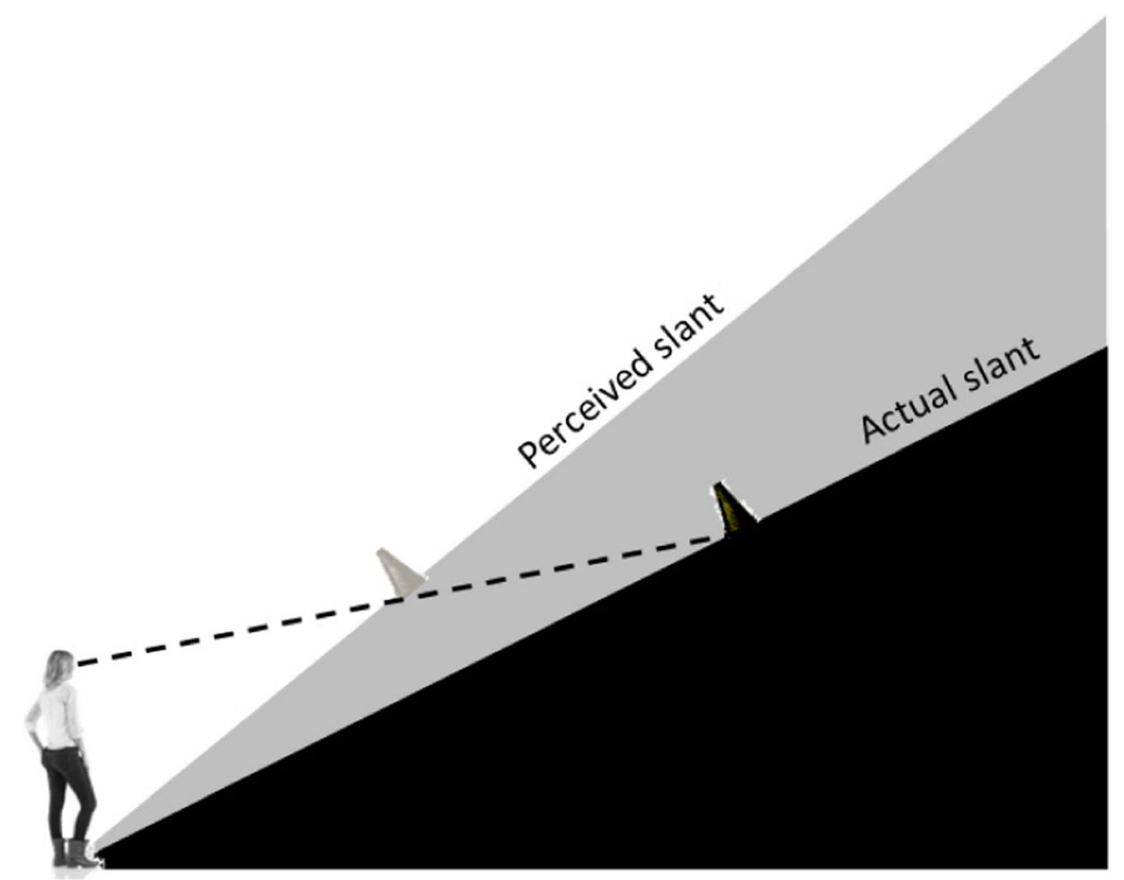

Fig. 15 How perceiving hill slant as steeper should lead to perceive a cone on the hill as closer. Note that the cone on the perceived slant ( $g r e y$ cone) is at a closer distance than the cone on the actual slant (black cone). Adapted from Stefanucci et al. (2005) 
multiple conditions. This leads to increased variability, and is thus likely to produce difference scores that do not have adequate reliability to do research on individual differences. As far as we know, the visual matching measure to assess the distance-on-hills effect is the only method to evaluate an action-specific effect that has good intrasubject reliability. Consequently, this particular method is poised to be useful for making important theoretical implications for the actionspecific approach because it is the only known paradigm that can be used to assess contributions of individual characteristics regarding these effects. Future studies aiming to determine the mechanism of the distance-on-hill effect by exploring individual differences should only use the visual matching task.

Taken together, these studies provide further evidence to suggest that the perceived elongation of distances on a hill is pervasive and perceptual. The finding supports the larger claim of the action-specific account that a perceiver's ability to act influences spatial perception (Witt, 2011, in press; Witt et al., 2016). Given that action's influence on perception would denote a top-down effect wherein perception is influenced by factors other than optical information (Firestone \& Scholl, 2016), it is critical to determine whether purported effects are truly perceptual. Here, we found converging evidence across multiple measures including an action-based measure. This convergence suggests a common, underlying effect in perception as opposed to response-specific processes involved in generating the response (Foley, 1977; Philbeck \& Loomis, 1997; Philbeck \& Witt, 2015). The current studies further advance the claim that perceivers see the spatial layout of the environment in terms of their ability to act within it.

Author note Nathan L. Tenhundfeld and Jessica K. Witt, Department of Psychology, Colorado State University, Fort Collins, CO 80523.

The author would like to thank Isabel Gauthier for her assistance with issues related to reliability and Nicole Versace for her drawings. This work was supported by grants from the National Science Foundation to J.K.W. (BCS-1348916 and BCS-1632222).

\section{References}

Bhalla, M., \& Proffitt, D. R. (1999). Visual-motor recalibration in geographical slant perception. Journal of Experimental Psychology: Human Perception and Performance, 25(4), 1076.

Cohen, J. (2013). Statistical power analysis for the behavioral sciences. New York, NY: Academic Press.

Cutting, J. E., \& Vishton, P. M. (1995). Perceiving layout and knowing distance: The integration, relative potency, and contextual use of different information about depth. In W. Epstein \& S. Rogers (Eds.), Perceiving space and motion (pp. 69-117). London, UK: Academic Press.

Durgin, F. H., Baird, J. A., Greenburg, M., Russell, R., Shaughnessy, K., \& Waymouth, S. (2009). Who is being deceived? The experimental demands of wearing a backpack. Psychonomic Bulletin \& Review, 16(5), 964-969.
Firestone, C., \& Scholl, B. J. (2016). Cognition does not affect perception: Evaluating the evidence for 'top-down' effects. The Behavioral and Brain Sciences, 39, e229.

Foley, J. M. (1977). Effect of distance information and range on two indices of visually perceived distance. Perception, 6(4), 449-460.

Franzen, M. D., Tishelman, A. C., Sharp, B. H., \& Friedman, A. G. (1987). An investigation of the test-retest reliability of the Stroop color-word test across two intervals. Archives of Clinical Neuropsychology, 2(3), 265-272.

Gogel, W. C. (1990). A theory of phenomenal geometry and its applications. Perception \& Psychophysics, 48(2), 105-123.

Goodale, M. A., \& Milner, A. D. (1992). Separate visual pathways for perception and action. Trends in Neurosciences, 15(1), 20-25.

Guettling, T. P., Park, S. Y., Kenemans, J. L., \& Neggers, S. F. W. (2013). TMS of the anterior intraparietal area selectively modulates orientation change detection during action preparation. Journal of Neurophysiology, 110(1), 33-41.

Heath, M., Rival, C., Westwood, D. A., \& Neely, K. (2005). Time course analysis of closed- and open-loop grasping of the Müller-Lyer illusion. Journal of Motor Behavior, 37(3), 179-185.

Lessard, D. A., Linkenauger, S. A., \& Proffitt, D. R. (2009). Look before you leap: Jumping ability affects distance perception. Perception, 38(12), 1863.

Li, Z., \& Durgin, F. H. (2012). Manual matching of perceived surface orientation is affected by arm posture: Evidence of calibration between proprioception and visual experience in near space. Experimental Brain Research, 216(2), 299-309.

Linkenauger, S. A., Lerner, M. D., Ramenzoni, V. C., \& Proffitt, D. R. (2012). A perceptual-motor deficit predicts social and communicative impairments in individuals with autism spectrum disorders. Autism Research, 5(5), 352-362. doi:10.1002/Aur.1248

Loomis, J. M. (2016). Proposed applications of research on actionspecific effects are premature. Journal of Applied Research in Memory and Cognition, 1(5), 77-79.

Loomis, J. M., \& Philbeck, J. W. (2008). Measuring spatial perception with spatial updating and action. In Carnegie Symposium on Cognition, 2006, Pittsburgh, PA, US: Psychology Press.

Loomis, J. M., Da Silva, J. A., Fujita, N., \& Fukusima, S. S. (1992). Visual space perception and visually directed action. Journal of Experimental Psychology: Human Perception and Performance, 18(4), 906.

MacLennan, R. N. (1993). Interrater reliability with SPSS for Windows 5.0. The American Statistician, 47(4), 292-296.

Open Science Collaboration. (2015). Estimating the reproducibility of psychological science. Science, 349(6251), aac4716.

Pagano, C. C., \& Isenhower, R. W. (2008). Expectation affects verbal judgments but not reaches to visually perceived egocentric distances. Psychonomic Bulletin \& Review, 15(2), 437-442.

Philbeck, J. W., \& Loomis, J. M. (1997). Comparison of two indicators of perceived egocentric distance under full-cue and reduced-cue conditions. Journal of Experimental Psychology: Human Perception and Performance, 23(1), 72.

Philbeck, J. W., \& Witt, J. K. (2015). Action-specific influences on perception and post-perceptual processes: Present controversies and future directions. Psychological Bulletin, 141, 1120-1144.

Proffitt, D. R. (2006). Embodied perception and the economy of action. Perspectives on Psychological Science, 1(2), 110-122.

Proffitt, D. R., Bhalla, M., Gossweiler, R., \& Midgett, J. (1995). Perceiving geographical slant. Psychonomic Bulletin \& Review, 2(4), 409-428.

Proffitt, D. R., Stefanucci, J., Banton, T., \& Epstein, W. (2003). The role of effort in perceiving distance. Psychological Science, 14(2), 106112.

Poulton, E. C. (1979). Models for biases in judging sensory magnitude. Psychological Bulletin, 86(4), 777. 
Richler, J. J., Floyd, R. J., \& Gauthier, I. (2014). The Vanderbilt Holistic Face Processing Test: A short and reliable measure of holistic face processing. Journal of Vision, 14, 1-14.

Simonsohn, U., Nelson, L. D., \& Simmons, J. P. (2014). P-curve: A key to the file-drawer. Journal of Experimental Psychology: General, 143(2), 534-547.

Sinai, M. J., Ooi, T. L., \& He, Z. J. (1998). Terrain influences the accurate judgement of distance. Nature, 395(6701), 497-500.

Stefanucci, J. K., \& Proffitt, D. R. (2009). The roles of altitude and fear in the perception of height. Journal of Experimental Psychology: Human Perception and Performance, 35(2), 424-438.

Stefanucci, J. K., Proffitt, D. R., Banton, T., \& Epstein, W. (2005). Distances appear different on hills. Perception \& Psychophysics, 67(6), 1052-1060.

Stevens, S. S. (1957). On the psychophysical law. Psychological Review, 64(3), 153.

Strauss, G. P., Allen, D. N., Jorgensen, M. L., \& Cramer, S. L. (2005). Test-retest reliability of standard and emotional Stroop tasks an investigation of color-word and picture-word versions. Assessment, 12(3), 330-337.

Sugovic, M., \& Witt, J. K. (2013). An older view on distance perception: Older adults perceive walkable extents as farther. Experimental Brain Research, 226(3), 383-391.

Taylor-Covill, G. A., \& Eves, F. F. (2013). Slant perception for stairs and screens: Effects of sex and fatigue in a laboratory environment. Perception, 42(4), 459-469.

Tucker, M., \& Ellis, R. (1998). On the relations between seen objects and components of potential actions. Journal of Experimental Psychology: Human Perception and Performance, 24(3), 830.
Witt, J. K. (2011). Action's effect on perception. Current Directions in Psychological Science, 20(3), 201-206.

Witt, J. K. (in press). Action Potential Influences Spatial Perception: Evidence for Genuine Top-Down Effects on Perception. Psychonomic Bulletin \& Review. doi:10.3758/s13423-016-1184-5

Witt, J. K., Kemmerer, D., Linkenauger, S. A., \& Culham, J. (2010). A functional role for motor simulation in identifying tools. Psychological Science, 21(9), 1215-1219. doi:10.1177/ 0956797610378307

Witt, J. K., Proffitt, D. R., \& Epstein, W. (2004). Perceiving distance: A role of effort and intent. Perception, 33, 577-590.

Witt, J. K., Proffitt, D. R., \& Epstein, W. (2005). Tool use affects perceived distance, but only when you intend to use it. Journal of Experimental Psychology: Human Performance and Perception, 31(5), 880.

Witt, J. K., Proffitt, D. R., \& Epstein, W. (2010). When and how are spatial perceptions scaled? Journal of Experimental Psychology: Human Perception and Performance, 36(5), 1153-1160. doi:10. 1037/A0019947

Witt, J. K., Sugovic, M., Tenhundfeld, N. L., \& King, Z. R. (2016). An action-specific effect on perception that survives all pitfalls. Behavioral and Brain Sciences, 39, e261.

Woods, A. J., Philbeck, J. W., \& Danoff, J. V. (2009). The various perceptions of distance: An alternative view of how effort affects distance judgments. Journal of Experimental Psychology: Human Perception and Performance, 35(4), 1104-1117.

Yang, S. J., \& Beilock, S. L. (2011). Seeing and doing: Ability to act moderates orientation effects in object perception. The Quarterly Journal of Experimental Psychology, 64(4), 639-648. 